PROCEEDINGS OF THE

AMERICAN MATHEMATICAL SOCIETY

Volume 134, Number 7 , Pages 1985-1993

S 0002-9939(05)08213-4

Article electronically published on December 19, 2005

\title{
AN EXAMPLE OF APPLICATION OF THE NIELSEN THEORY TO INTEGRO-DIFFERENTIAL EQUATIONS
}

\author{
JAN ANDRES AND TOMÁŠ FÜRST \\ (Communicated by Carmen C. Chicone)
}

\begin{abstract}
A new nontrivial example of an application of the Nielsen fixedpoint theory is presented, this time, to integro-differential equations. The emphasis is on the parameter space so that no subdomain becomes invariant under the related solution (Hammerstein) operator. Thus, at least three (harmonic) periodic solutions are established to a planar integro-differential system.
\end{abstract}

\section{INTRODUCTION}

The Nielsen theory allows us to obtain the lower estimate of the number of fixed points. More precisely, if $f: X \rightarrow X$ is a compact (continuous) map on a (metric) ANR-space $X$, then a nonnegative integer $N(f)$, called the Nielsen number of $f$, is defined such that (see e.g. [6]):

- $N(f) \leq \# \operatorname{Fix}(f):=\operatorname{card}\{\hat{x} \in X: f(\hat{x})=\hat{x}\}$,

- $N(f)=N(\tilde{f})$, for any compact $\tilde{f}: X \rightarrow X$ which is compactly homotopic to $f$, i.e. if there is a compact map $h: X \times[0,1] \rightarrow X$ such that $h_{0}=f$, $h_{1}=\tilde{f}$, where $h_{t}(x):=h(x, t)$, for $t \in[0,1]$.

Let us recall that by an $A N R$-space $X$, we understand that, for any metric space $Y$, its closed subset $S \subset Y$ and a continuous mapping $g: S \rightarrow X$, there exists a (continuous) extension of $g$ onto some neighbourhood of $S$ in $Y$. Roughly speaking, ANR-spaces are, up to retractions and up to homeomorphic images, open subsets of normed spaces.

Given a compact $f: X \rightarrow X$ on $X \in \mathrm{ANR}$, we say that $x, y \in \operatorname{Fix}(f)$ are Nielsen related if there exists a path $u:[0,1] \rightarrow X$ such that $u(0)=x, u(1)=y$, and $u$, $f(u)$ are homotopic, keeping the endpoints fixed. Since the Nielsen relation is an equivalence (cf. [6]), $\operatorname{Fix}(f)$ splits into (in view of compactness, a finite number of) fixed-point classes.

If, for a Nielsen class $\mathcal{N} \subset \operatorname{Fix}(f)$, we have $\operatorname{ind}(\mathcal{N}, f) \neq 0$, i.e. if the associated fixed-point index is nontrivial, then $\mathcal{N}$ is called essential. The Nielsen number $N(f)$ is then defined to be the number of essential Nielsen classes. For more details, see e.g. 1, [3], 6], 7].

Received by the editors January 18, 2005 and, in revised form, February 8, 2005.

2000 Mathematics Subject Classification. Primary 34C25, 47H10, 54H25.

Key words and phrases. Nielsen number, lower estimate of fixed points, multiplicity results, integro-differential equations, nontrivial application.

This work was supported by the Council of Czech Government (MSM 6198959214).

(C)2005 American Mathematical Society Reverts to public domain 28 years from publication 1985 
A nontrivial application of the Nielsen theory to differential equations is always a difficult task. This problem, associated with the name of J. Leray, has not yet been solved in a satisfactory way. There are some contributions (for the survey of results in this field, see [2], 7], 12]), but practically all of them have some handicap.

In [1, we tried to construct such a nontrivial example for a planar differnetial system without implemented (or small) parameters, but as observed in [10, there was a gap concerning assumptions imposed on the related solution operator in the parameter space. This gap can be simply avoided by adding some additional restricitons (see Theorem 6.17 on p. 359 in [3]), but then some subdomains of the parameter space become invariant under the solution operator, and so alternative approach (rather than the Nielsen theory) can be used, even in a more efficient way (cf. [10]). The subdomain subinvariance is also present in [8], 9], where integral equations involving Urysohn-type operators are treated for the same goal.

In the present paper, we would like to avoid this drawback. Our aim is to present a multiplicity criterium for solutions of a planar integro-differential system, by means of the Nielsen number, in as transparent a way as possible. Thus, the right-hand sides can apparently take a more general form, their regularity can be weakened and the multiplicity criteria in terms of inequalities can be improved. This is only indicated in the concluding remarks.

\section{Applied Method}

Before constructing the desired example, we develop, by means of the Nielsen number, a simple technique for studying a rather general class of operator equations with constraints. Although it is only a slight modification of a very special case of multivalued boundary value problems studied in the monograph [3] (cf. Theorem 1.25 on p. 242 and Theorem 6.4 on p. 351 in [3]), we shall prove it fully, for the sake of completeness.

Let $K \subset \mathbb{R}$ be a compact interval and let $C$ denote the set of all continuous functions from $K$ to $\mathbb{R}^{n}$. Let $\|\cdot\|$ denote the usual maximal norm on $C$. Let us deal with a general operator equation

$$
x^{\prime}=\mathcal{G}_{\lambda}(x),
$$

where $\lambda \in[0,1]$, together with a constraint (e.g. a boundary or initial condition)

$$
x \in S
$$

where the properties of operator $\mathcal{G}_{\lambda}: C \rightarrow C$ and set $S \subset C$ are to be specified later. To keep this presentation simple and self-contained, we shall consider classical solutions to (1), (2), i.e. solutions $x \in C^{1}$ which satisfy (11), for all $t \in K$. We start with the following lemma describing the properties of the solution operator to the fully linearized system (1).

Lemma 1. Let $\mathcal{G}_{\lambda}: C \times[0,1] \rightarrow C, \lambda \in[0,1]$, be a bounded and continuous operator. Let $S \subset C$ be bounded. Let there exist a closed and bounded set $Q \subset C$ such that, for each $q \in Q$, the equation

$$
x^{\prime}=\mathcal{G}_{\lambda}(q)
$$

together with (2) has, for each $\lambda \in[0,1]$, a unique solution. Let us denote by $T: Q \times[0,1] \rightarrow S$ the solution operator which takes any $(q, \lambda) \in Q \times[0,1]$ to the unique solution of (3), (2). Then $T$ is compact (and continuous). 
Proof. Let us first show the compactness of $\overline{T(Q \times[0,1])}$. Let $T(Q \times[0,1])$ be bounded, because so is $S$. In view of the Arzelà-Ascoli lemma, it is sufficient to show that $T(Q \times[0,1])$ is equi-continuous. Let us take an arbitrary $(q, \lambda) \in Q \times[0,1]$ and let $x=T(q, \lambda)$. Since $x$ is a solution to (3), it is continuously differentiable. We thus have

$$
\begin{aligned}
\left|x\left(t_{1}\right)-x\left(t_{2}\right)\right| & =\left|\int_{t_{1}}^{t_{2}} x^{\prime}(s) d s\right|=\left|\int_{t_{1}}^{t_{2}} \mathcal{G}_{\lambda}(q(s)) d s\right| \leq \int_{t_{1}}^{t_{2}}\left|\mathcal{G}_{\lambda}(q(s))\right| d s \\
\leq \int_{t_{1}}^{t_{2}}\left\|\mathcal{G}_{\lambda}(q)\right\| d s & \left.\leq C_{\mathcal{G}}\|q\|\left|t_{2}-t_{1}\right| \leq \quad \text { (by boundedness of } \mathcal{G}_{\lambda}\right) \\
& \leq M\|q\|\left|t_{2}-t_{1}\right| \leq \quad \text { (by boundedness of } Q \text { ) } \\
& \leq M N\left|t_{1}-t_{2}\right|,
\end{aligned}
$$

where $C_{\mathcal{G}}$ is a nonnegative constant. This proves the equi-continuity of $T(Q \times[0,1])$.

Let us now prove the continuity of $T$. In view of the compactness of $\overline{T(Q \times[0,1])}$, it is sufficient to prove that $T$ has a closed graph (see e.g. Proposition 3.16 on p. 33 in 3]). Let us consider a sequence $\left\{q_{k}, \lambda_{k}, x_{k}\right\}$ in the graph of $T$ such that $q_{k} \rightarrow q$ in $C, \lambda_{k} \rightarrow \lambda$, and $x_{k} \rightarrow x$ in $C$. We want to show that $x=T(q, \lambda)$, which means that $x^{\prime}=\mathcal{G}_{\lambda}(q)$. Since $q_{k} \in Q$ and $Q$ is closed, we have $q \in Q$. Relation $x_{k}=T\left(q_{k}, \lambda_{k}\right)$ means that $x_{k}^{\prime}=\mathcal{G}_{\lambda_{k}}\left(q_{k}\right)$, and consequently

$$
\left\|x_{k}^{\prime}\right\|=\left\|\mathcal{G}_{\lambda}\left(q_{k}\right)\right\| \leq C_{\mathcal{G}}\left\|q_{k}\right\| \leq M N
$$

which shows that $\left|x_{k}^{\prime}(t)\right| \leq \alpha(t)$, where $\alpha \in L^{1}(K)$. Moreover, let $\left\{x_{k}(t)\right\}$ be bounded, for each $t \in K$, because the sequence $x_{k}$ is convergent in $C$. This implies that (cf. Theorem 4 on pp. 13-14 in 4]) we have a selected subsequence, denoted just the same, such that $x_{k} \rightarrow x$, uniformly on $K$, and $x_{k}^{\prime} \rightarrow x^{\prime}$, weakly in $L^{1}(K)$. On the other hand, we have $x_{k}^{\prime}=\mathcal{G}_{\lambda_{k}}\left(q_{k}\right) \rightarrow \mathcal{G}_{\lambda}(q)$ in $C \times[0,1]$ which implies that $x_{k}^{\prime} \rightarrow \mathcal{G}_{\lambda}(q)$, weakly in $L^{1}(K)$. The uniqueness of the weak limit gives us $x^{\prime}=\mathcal{G}_{\lambda}(q)$ in $L^{1}(K)$, and since both sides are continuous, $x^{\prime}=\mathcal{G}_{\lambda}(q)$ in $C$, which proves that $T$ has a closed graph.

As already pointed out, if $X$ is an ANR-space and $f: X \rightarrow X$ is a compact (continuous) map, we can define the Nielsen number $N(f)$ which provides the lower estimate for the number of fixed points of $f$ in $X$. Moreover, $N(f)$ is a homotopy invariant in the sense that any (compact) $\tilde{f}: X \rightarrow X$ compactly homotopic to $f$ has at least $N(f)$ fixed points in $X$. We can thus state the following proposition.

Proposition 1. Let the assumptions of Lemma 1 be satisfied. Let $Q$ be an ANRspace and let $\overline{T(Q \times[0,1])} \subset Q \cap S$. Then, for each $\lambda \in[0,1]$, problem (11), (2) has at least $N(\tilde{T})$ solutions in $Q$, where $\tilde{T} \sim T$ is any mapping, compactly homotopic to $T$.

Proof. Each fixed point of $T$ is a solution to (11), (2). Since $Q$ is an ANR-space and $T$ is a compact continuous selfmap of $Q$, the Nielsen number $N(T)$ is welldefined and gives the lower estimate of the number of fixed points of any compact $\tilde{T}$, compactly homotopic to $T$. 


\section{Nontrivial example}

Let $x_{i}:[0, \omega] \rightarrow \mathbb{R}$, for $i=1,2, x=\left(x_{1}, x_{2}\right), \varphi \in\left[0, \frac{\pi}{4}\right], a>0$, and consider the following system of integro-differential equations:

$$
\begin{aligned}
x_{1}^{\prime}+a x_{1} & =\sqrt[3]{p_{2}(x)} \cos \varphi-\sqrt[3]{p_{1}(x)} \sin \varphi+\varphi e, \\
x_{2}^{\prime}+a x_{2} & =\sqrt[3]{p_{1}(x)} \cos \varphi+\sqrt[3]{p_{2}(x)} \sin \varphi+\varphi e
\end{aligned}
$$

where $e:[0, \omega] \rightarrow \mathbb{R}$ is a continuous function with $|e(t)| \leq E$, for all $t \in[0, \omega]$, and

$$
p_{i}(x)=\frac{1}{\omega} \int_{0}^{\omega} x_{i}(s) d s-B\left(\frac{1}{\omega} \int_{0}^{\omega} x_{i}(s) d s-x_{i}\right),
$$

with $B>0$. For $\varphi=\frac{\pi}{4}$, the system takes the form

$$
\begin{aligned}
& x_{1}^{\prime}+a x_{1}=\frac{\sqrt{2}}{2}\left(\sqrt[3]{p_{2}(x)}-\sqrt[3]{p_{1}(x)}\right)+\frac{\pi}{4} e, \\
& x_{2}^{\prime}+a x_{2}=\frac{\sqrt{2}}{2}\left(\sqrt[3]{p_{1}(x)}+\sqrt[3]{p_{2}(x)}\right)+\frac{\pi}{4} e
\end{aligned}
$$

while, for $\varphi=0$, it reduces to

$$
x_{1}^{\prime}+a x_{1}=\sqrt[3]{p_{2}(x)}, \quad x_{2}^{\prime}+a x_{2}=\sqrt[3]{p_{1}(x)} .
$$

We shall be looking for the lower estimate of the number of $\omega$-periodic solutions to (6), (77). Let us denote $\lambda=\frac{4 \varphi}{\pi}$ and define $\mathcal{G}_{\lambda}: C\left([0, \omega], \mathbb{R}^{2}\right) \times[0,1] \rightarrow C\left([0, \omega], \mathbb{R}^{2}\right)$ by

$$
\begin{aligned}
\mathcal{G}_{\lambda}:= & \left(-a x_{1}+\sqrt[3]{p_{2}(x)} \cos \varphi-\sqrt[3]{p_{1}(x)} \sin \varphi+\varphi e,\right. \\
& \left.-a x_{2}+\sqrt[3]{p_{1}(x)} \cos \varphi+\sqrt[3]{p_{2}(x)} \sin \varphi+\varphi e\right)
\end{aligned}
$$

Then $\mathcal{G}_{\lambda}$ satisfies the assumptions of Lemma 1 which will be used to obtain at least two $\omega$-periodic solutions to (4), (5).

Let us define sets $S=Q \subset \mathcal{C}\left([0, \omega], \mathbb{R}^{2}\right)$ as follows. Function $q=\left(q_{1}, q_{2}\right)$ belongs to $Q$ if the following conditions are satisfied:

(i) $q(0)=q(\omega)$ ( $\omega$-periodicity),

(ii) $|q(t)| \leq R$, for all $t \in[0, \omega]$ (boundedness),

(iii) $\left|q_{1}(t)\right| \geq \delta$ or $\left|q_{2}(t)\right| \geq \delta$, for all $t \in[0, \omega]$ (uniform boundedness of one component from below),

(iv) $q(t)=\bar{q}+\tilde{q}(t)$, where $\bar{q}:=\frac{1}{\omega} \int_{0}^{\omega} q(s) d s$ is the integral average of $q$ on $[0, \omega]$ (thus, $\frac{1}{\omega} \int_{0}^{\omega} \tilde{q}(s) d s=0$ ) and $|\tilde{q}(t)| \leq \varepsilon$, for all $t \in[0, \omega]$ (function $q$ differs from its integral average by less than $\varepsilon$ ).

The values of $a$ and $\omega$ having been given, we shall specify the values of $B, \delta, R, E$ and $\varepsilon$ in the subsequent parts.

It follows from Corollary 4.4 on p. 284 in [13] (cf. 5]) that a finite union of closed convex sets in a Banach space is an ANR-space. Since $Q$ can be regarded as a union of four closed convex sets in $C\left([0, \omega], \mathbb{R}^{2}\right)$, it must be an ANR-space.

For the homotopic parameter $\varphi=0$, system (4), (5) reduces to a simpler case, which can be easily handled (in fact, we can explicitely compute two constant fixed points). For $\varphi=\frac{\pi}{4}$, the situation becomes nontrivial. We shall show that set $Q$ is invariant under the solution operator, which takes a parameter $q \in Q$ to the solution $x$ of the linearized equation. In this case, no obvious or easily detectable subset of $Q$ can be recognized to be separately invariant. 
In order to apply Proposition 1, we use the method of Schauder linearization. Let us take an arbitrary $q \in Q$. The system of fully linearized equations takes the form

$$
\begin{aligned}
x_{1}^{\prime}+a x_{1} & =\sqrt[3]{p_{2}(q)} \cos \varphi-\sqrt[3]{p_{1}(q)} \sin \varphi+\varphi e \\
x_{2}^{\prime}+a x_{2} & =\sqrt[3]{p_{1}(q)} \cos \varphi+\sqrt[3]{p_{2}(q)} \sin \varphi+\varphi e
\end{aligned}
$$

where

$$
p_{i}(q)=\frac{1}{\omega} \int_{0}^{\omega} q_{i}(s) d s-B\left(\frac{1}{\omega} \int_{0}^{\omega} q_{i}(s) d s-q_{i}\right),
$$

for $i=1,2$.

Denoting $\bar{q}:=\frac{1}{\omega} \int_{0}^{\omega} q(s) d s$ as the integral average of $q$ on $[0, \omega]$, we can write $p: Q \subset \mathcal{C}\left([0, \omega], \mathbb{R}^{2}\right) \rightarrow \mathcal{C}\left([0, \omega], \mathbb{R}^{2}\right)$ in the form

$$
p(q)=\bar{q}-B(\bar{q}-q) .
$$

For $B=1$, operator $p$ reduces to identity. For $B<1$, the operator "shrinks" function $q$ closer to its integral average. Indeed, if $q=\bar{q}+\tilde{q}$, where $|\tilde{q}(t)| \leq \varepsilon$, for all $t \in[0, \omega]$, then operator $p$ takes $q$ to $p(q)=\bar{q}-B \tilde{q}$.

The fully linearized system (8), (9) posesses, for any $q \in Q$, a unique solution $x(t)$ which is given by the known convolution with the Green operator

$$
x_{i}(t)=\int_{0}^{\omega} G(t, s) f_{i}(s) d s,
$$

where $f_{i}$ stand for the right-hand side of equations (8), (9), namely,

$$
\begin{aligned}
& f_{1}(s):=\sqrt[3]{p_{2}(q)} \cos \varphi-\sqrt[3]{p_{1}(q)} \sin \varphi+\varphi e \\
& f_{2}(s):=\sqrt[3]{p_{1}(q)} \cos \varphi+\sqrt[3]{p_{2}(q)} \sin \varphi+\varphi e
\end{aligned}
$$

Let us denote by $T_{\varphi}$ the solution operator which takes $q \in Q$ to the unique solution $x$ of the linearized system given by (12). We shall prove that $Q$ is invariant under $T_{\varphi}$, namely that $x=T_{\varphi}(q) \in Q$, for each $q \in Q$.

Let us take $q \in Q$ arbitrary. Operator $p$ defined by (11) takes $q$ to $p(q)$ such that $p(q)=\bar{q}+\tilde{p}$, where $|\tilde{p}(t)| \leq B \varepsilon$. Substituting this $p(q)$ into (13) and (14), we obtain $f_{i}(t)=F_{i}(t)+\tilde{f}(t)$, where

$$
F_{1}:=\sqrt[3]{\bar{q}_{2}} \cos \varphi-\sqrt[3]{\bar{q}_{1}} \sin \varphi, \quad F_{2}:=\sqrt[3]{\bar{q}_{1}} \cos \varphi+\sqrt[3]{\bar{q}_{2}} \sin \varphi,
$$

and $\left|\tilde{f}_{i}(t)\right| \leq 3 \sqrt[3]{B \varepsilon}+\frac{\pi E}{4}$ for all $t \in[0, \omega]$. This estimate can be shown as follows: for $\left|\bar{q}_{i}\right| \geq 1$, one can show by direct calculation that $\left|\sqrt[3]{\bar{q}_{i}+\tilde{p}_{i}}-\sqrt[3]{\bar{q}_{i}}\right| \leq \sqrt[3]{B \varepsilon}$, provided that $\left|\tilde{p}_{i}\right| \leq B \varepsilon \leq 1$. For $\left|\bar{q}_{i}\right| \leq 1$, a careful examination of function $\left|\sqrt[3]{\bar{q}_{i}+\tilde{p}_{i}}-\sqrt[3]{\bar{q}_{i}}\right|$ reveals that it is bounded from above by value $2 \frac{2}{3} \sqrt[3]{B \varepsilon}$, provided again that $\left|\tilde{p}_{i}\right| \leq B \varepsilon \leq 1$. Altogether, $f_{i}$ differs from $F_{i}$ not more than

$$
2 \frac{\sqrt{2}}{2} 2^{\frac{2}{3}} \sqrt[3]{B \varepsilon}+\frac{\pi E}{4} \leq 3 \sqrt[3]{B \varepsilon}+\frac{\pi E}{4} .
$$

The unique solution of the linearized problem is given by (12), where

$$
G(t, s)= \begin{cases}\frac{1}{1-e^{-a \omega}} e^{-a t} e^{a s}, & \text { for } 0 \leq s \leq t, \\ \frac{1}{1-e^{-a \omega}} e^{-a t} e^{-a \omega} e^{a s}, & \text { for } t \leq s \leq \omega .\end{cases}
$$


Substituting (15) and (16) into (12), we obtain $x_{i}(t)=\frac{F_{i}}{a}+\tilde{x}_{i}(t)$, where $\tilde{x}_{i}$ satisfies the inequality

$$
\left|\tilde{x}_{i}(t)\right| \leq \frac{3 \sqrt[3]{B \varepsilon}+\frac{\pi}{4} E}{a},
$$

for all $t \in[0, \omega]$. We can now take $B$ and $E$ small enough to fulfil

$$
\frac{\sqrt{2}\left(3 \sqrt[3]{B \varepsilon}+\frac{\pi}{4} E\right)}{a} \leq \frac{\varepsilon}{2}, \quad \text { for example } B \leq\left(\frac{a}{12}\right)^{3} \frac{\varepsilon^{2}}{\sqrt{2}} \text { and } E \leq \frac{a \varepsilon}{\pi \sqrt{2}} .
$$

This means that function $x$ differs from a constant function by less than $\frac{\varepsilon}{2}$, which implies that it differs from its integral average by less than $\varepsilon$. The above calculations ensure that the solution $x=T_{\varphi}(q)$ satisfies condition (iv) of the definition of the parameter set $Q$, independently on $q \in Q$ and $\varphi \in\left[0, \frac{\pi}{4}\right]$. Condition (i) is trivially satisfied by the form of the Green function $G$.

We must further ensure that, for each $q \in Q$, function $x=T_{\varphi}(q)$ satisfies conditions (ii) and (iii). Since both $q$ and $x=T_{\varphi}(q)$ differ from their integral averages by less than $\varepsilon$, let us first deal with constant functions. This is easy, because the solution mapping $T_{\varphi}$ takes constant functions to functions that differ from a constant function by less than $\frac{\pi E}{4 a}$, and it is a composition of

- reflection $\left(\bar{q}_{1}, \bar{q}_{2}\right) \rightarrow\left(\bar{q}_{2}, \bar{q}_{1}\right)$,

- re-scaling $\left(\bar{q}_{1}, \bar{q}_{2}\right) \rightarrow \frac{1}{a}\left(\sqrt[3]{\bar{q}_{1}}, \sqrt[3]{\bar{q}_{2}}\right)$,

- rotation $\left(\bar{q}_{1}, \bar{q}_{2}\right) \rightarrow\left(\bar{q}_{1} \cos \varphi-\bar{q}_{2} \sin \varphi, \bar{q}_{2} \cos \varphi+\bar{q}_{1} \sin \varphi\right)$

by angle $\varphi$ in the anti-clockwise direction.

The re-scaling part of the composition ensures that constants $R$ and $\delta$ can be specified so that the solution operator $T_{\varphi}$ takes constant functions satisfying (ii) and (iii) to functions that again satisfy (ii) and (iii). Since functions in $Q$ differ from their integral averages by less than $\varepsilon$, we need to find $R, \delta$ and $\varepsilon$ such that the following conditions are satisfied:

$$
\frac{1}{a} \sqrt[3]{R} \leq R-\varepsilon-\frac{\pi E}{4 a} \quad \text { and } \quad \frac{\sqrt{2}}{2 a} \sqrt[3]{\delta} \geq \delta+\sqrt{2} \varepsilon+\frac{\pi E}{4 a} .
$$

Inequalities (18) guarantee that $T_{\varphi}(q)$ satisfies conditions (ii) and (iii) of the definition of the parameter set $Q$. Taking further $\varepsilon \leq \frac{\delta}{2}$ ensures that $Q$ is a nontrivial ANR-space (leaving the "hole" inside).

Starting from $a>0$ and $\omega>0$, we have specified constants $R, \delta, B, E$ and $\varepsilon$ such that set $Q$ becomes invariant under the solution operator $T_{\varphi}$ which takes any $q \in Q$ to the solution $x$ of the linearized problem (8), (9), for $\varphi \in\left[0, \frac{\pi}{4}\right]$. Moreover, observe that, for $\varphi=\frac{\pi}{4}$, there are no easily detectable subdomains of $Q$ separately invariant under operator $T_{\frac{\pi}{4}}$. Figure 1 shows how operator $T_{\frac{\pi}{4}}$ treats constant functions in $Q$, for a particular choice of $R$ and $\delta$, and helps understand why we cannot easily detect any subinvariant domains of $Q$.

Since $Q$ is invariant under $T_{\varphi}$, for any $\varphi \in\left[0, \frac{\pi}{4}\right]$, Proposition 1 ensures that system (4), (5) admits at least $N\left(T_{\frac{\pi}{4}}\right)$ solutions. Since $T_{\frac{\pi}{4}}$ is compactly homotopic to $T_{0}$, we have $N\left(T_{\frac{\pi}{4}}\right)=N\left(T_{0}\right)$.

Let us further consider the retraction $r: Q \rightarrow Q$ which sends a function $q \in Q$ to its integral average $\bar{q}$. Let us define the homotopy $T^{\mu}:[0,1] \times Q \rightarrow Q$ by

$$
T^{\mu}(q):=\mu T_{0}(q)+(1-\mu) r\left(T_{0}(q)\right) .
$$



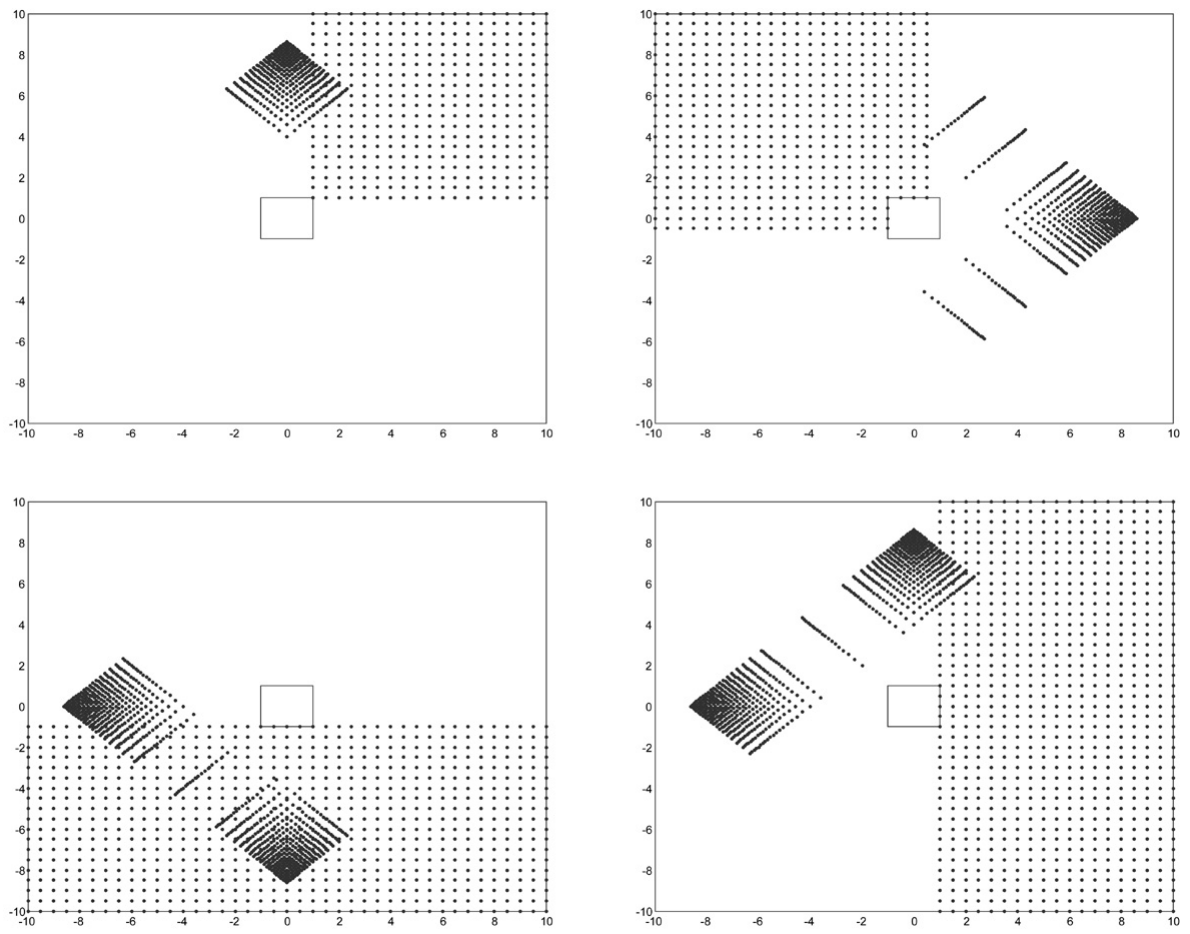

Figure 1. Behaviour of $T_{\frac{\pi}{4}}$ on constant functions on $Q$ for $R=10$, $\delta=1$ and $a=\frac{\sqrt{2}}{4}$. Rectangular grids of points represent constant functions $q \in Q$, the irregular grid represents their images under $T_{\frac{\pi}{4}}$. For simplicity, we take here $E=0$, so that the images of constant functions become constant again. No easily detectable regions of the domain are subinvariant.

This compact homotopy guarantees that $N\left(T^{1}\right)=N\left(T_{0}\right)$ equals $N\left(T^{0}\right)=N\left(r \circ T_{0}\right)$. We can thus restrict ourselves to the computation of $N\left(r \circ T_{0}\right)$. Let us denote by $\bar{Q}$ the subset of $Q$ consisting of constant functions. Since $r \circ T_{0}: Q \rightarrow \bar{Q}$, all the fixed points of $r \circ T_{0}$ have to belong to $\bar{Q}$. Let us therefore deal with the restriction $L:=\left.r \circ T_{0}\right|_{\bar{Q}}: \bar{Q} \rightarrow \bar{Q}$, which can be explicitly written in the form

$$
L\left(\bar{q}_{1}, \bar{q}_{2}\right):=\frac{1}{a}\left(\sqrt[3]{\bar{q}_{2}}, \sqrt[3]{\bar{q}_{1}}\right) .
$$

One can easily check by an explicit computation that $L$ has two fixed points in $\bar{Q}$ which belong to different Nielsen classes. Therefore, according to (reduction) Lemma 2 in [1], $N(L)=N\left(r \circ T_{0}\right)=2$. This finally shows that system (6), (7) admits at least two $\omega$-periodic solutions.

We are in the position to formulate the multiplicity criterium for $\omega$-periodic solutions to system (6), (7). 
Theorem 1. Let the following inequalities be satisfied:

$$
\delta<\frac{0.247}{a^{\frac{3}{2}}}, \quad \frac{\sqrt[3]{R}}{a} \leq R-\frac{5 \delta}{8}, \quad E \leq \frac{a \delta}{2 \pi \sqrt{2}}, \quad B \leq\left(\frac{a}{12}\right)^{3} \frac{\delta^{2}}{4 \sqrt{2}},
$$

and take $\varepsilon=\frac{\delta}{2}$. Then system (6), (77) admits at least three $\omega$-periodic solutions.

Proof. The assumptions guarantee that inequalities (17) and (18) are satisfied. Thus, two $\omega$-periodic solutions have already been obtained by means of the Nielsen number, as above. The third can be proved quite analogously to [1, by the additivity property of the fixed-point index, associated with the solution operator considered on $C\left([0, \omega], \mathbb{R}^{2}\right)$ with (i), (ii), (iv).

\section{Concluding Remarks}

Remark 1. The inequalities in Theorem 1 are satisfied e.g. for $a=\frac{\sqrt{2}}{4}, \delta=1$, $R=10, B \leq \frac{1}{2^{4} 3^{3}}$, and $E \leq \frac{1}{4 \pi}$, as in the example in Figure 1, where $E=0$.

Remark 2. Applying the Nielsen theory for admissible multivalued mappings as developed in [3, the same multiplicity criterium can be obtained e.g. for system (6), (7), where $e:[0, \omega] \times \mathbb{R}^{2} \rightarrow 2^{\mathbb{R}} \backslash\{\emptyset\}$ is an upper-Carathéodory (multivalued) mapping with convex and compact values such that $|e| \leq E$.

Remark 3. As far as we know, the only application of the Nielsen theory to integrodifferential equations was performed in a completely different way in Theorem 1 in 11. The multiplicity criterium is there, however, rather implicit, and so practically uncomparable to ours in Theorem 1 .

\section{REFERENCES}

1. J. Andres, A nontrivial example of application of the Nielses fixed-point theory to differential systems: problem of Jean Leray. Proceed. Amer. Math. Soc. 128 (2000), 2921-2931. MR 1664285 (2000m:47074)

2. J. Andres, Nielsen number and differential equations. Fixed Point Th. Appl. 2 (2005), 137167.

3. J. Andres and L. Górniewicz, Topological Fixed Point Principles for Boundary Value Problems. Kluwer, Dordrecht, 2003. MR.1998968 (2005a:47102)

4. J.-P. Aubin and A. Cellina, Differential Inclusions. Springer, Berlin, 1984. MR0755330 (85j:49010)

5. K. Borsuk, Theory of Retracts. Polish Sci. Publishers, Warsaw, 1967. MR0216473 (35:7306)

6. R. F. Brown, On the Nielsen fixed point theorem for compact maps. Duke Math. J. 36 (1969), 699-708. MR0250290 (40:3529)

7. R. F. Brown, More about Nielsen theories and their applications. In: "Handbook of Topological Fixed Point Theory" (R. F. Brown, M. Furi, L. Górniewicz and B. Jiang, eds.), Springer, Berlin, 2005, pp. 433-462. MR2171114

8. A. Yu. Borisovich, Z. Kucharski and W. Marzantowicz, Nielsen number and lower estimates for the number of solutions to a certain system of nonlinear integral equations. In: "New Developement in Global Analysis Series", Voronezh Univ. Press, Voronezh, 1994, pp. 3-10 (in Russian). MR 1321822 (96a:47103)

9. A. Yu. Borisovich, Z. Kucharski and W. Marzantowicz, A multiplicity result for a system of real integral equations by use of the Nielsen number. In: "Nielsen Theory and Reidemeister Torsion" (J. Jezierski, ed.), Banach Center Publ. vol. 49, Warsaw, pp. 9-18. MR1734721 (2000i:55004)

10. M. J. Capinski and K. Wójcik, Isolating segment for Carathéodory systems and existence for periodic solutions. Proceed. Amer. Math. Soc. 131, 8 (2003), 2443-2451. MR 1974642 (2003m:37019) 
11. M. Fečkan, Nielsen fixed point theory and nonlinear equations. J. Diff. Eqns 106, 2 (1993), 312-331. MR1251856 (94k:47093)

12. M. Fečkan, Multiple solutions of nonlinear equations via Nielsen fixed-point theory: a survey. In: "Nonlinear Analysis in Geometry and Topology" (T. M. Rassias, ed.), Hadronic Press, Palm Harbor, FL, 2000, pp. 77-97. MR1766782 (2001e:47095)

13. A. Granas and J. Dugundji, Fixed Point Theory, Springer, Berlin, 2003. MR 1987179 (2004d:58012)

Department of Mathematical Analysis, Faculty of Science, Palacký University, Tomkova 40, 77900 Olomouc-HejČín, Czech Republic

E-mail address: andres@inf.upol.cz

Department of Mathematical Analysis, Faculty of Science, Palacký University, Tomkova 40, 77900 Olomouc-HejČín, Czech Republic

E-mail address: tomas.furst@seznam.cz 